\title{
Antibacterial Effects of Afzelin Isolated from Cornus macrophylla on Pseudomonas aeruginosa, A Leading Cause of Illness in Immunocompromised Individuals
}

\author{
Sang Yeol Lee ${ }^{2}$, Young-Jin So ${ }^{1}$, Moon Sam Shin ${ }^{1,4}$, Jae Youl Cho ${ }^{3, *}$ and Jongsung Lee ${ }^{1,4, *}$
}

1 Department of Dermatological Health Management, College of Health Science, Eulji University, Seongna-Si, 461-713 Gyeonggi-Do, Korea; E-Mails: yjso@eulji.ac.kr (Y.-J.S.); msshin@eulji.ac.kr (M.S.S.)

2 Department of Life Science, Gachon University, San 65, Bokjeong-Dong, Sujeong-Gu, Seongnam-Si, 461-701 Gyeonggi-Do, Korea; E-Mail: leesaye@gachon.ac.kr

3 Department of Genetic Engineering, Sungkyunkwan University, Suwon-Si, 440-746 Gyeonggi-Do, Korea

4 Department of Senior Healthcare, BK21 plus program, Graduated school, Eulji University, Seongna-Si, 461-713 Gyeonggi-Do, Korea

* Authors to whom correspondence should be addressed; E-Mails: jaecho@skku.edu (J.Y.C.); jslee@eulji.ac.kr (J.L.); Tel.: +82-31-290-7868 (J.Y.C.); Fax: +82-31-290-7870 (J.Y.C.); Tel.: +82-31-740-7209 (J.L.); Fax: +82-31-740-7358 (J.L.).

Received: 7 February 2014; in revised form: 10 March 2014 / Accepted: 10 March 2014 / Published: 17 March 2014

\begin{abstract}
The crude ethyl acetate extract of the leaves of Cornus macrophylla showed antibacterial activity against Pseudomonas aeruginosa, a leading cause of illness in immunocompromised individuals. Bioactivity-guided separation led to the isolation of kaempferol 3-O- $\alpha$-L-rhamnopyranoside (afzelin). The structure was determined based on evaluation of its spectroscopic (UV, MS, and NMR) data. The minimum inhibitory concentration (MIC) of afzelin against Pseudomonas aeruginosa was found to be $31 \mu \mathrm{g} / \mathrm{mL}$. In addition, the results indicated that a hydroxyl group at $\mathrm{C} 3$ of the $\mathrm{C}$-ring of the flavone skeleton and the rhamnose group may act as a negative factor and an enhancing factor, respectively, in the antibacterial activities of afzelin.
\end{abstract}

Keywords: Cornus macrophylla; antibacterial activity; kaempferol 3-O- $\alpha$-Lrhamnopyranoside; Pseudomonas aeruginosa 


\section{Introduction}

P. aeruginosa is part of a large group of free-living bacteria that are ubiquitous in the environment. It can can cause a wide range of infections and is a leading cause of illness in immunocompromised individuals. In particular, this organism can be a serious pathogen in hospitals [1]. Specifically, it can cause endocarditis, osteomyelitis, pneumonia, urinary tract infections, gastrointestinal infections, keratitis, folliculitis, ear infections, and meningitis, and is a leading cause of septicemia. P. aeruginosa is also a major pathogen in burn and cystic fibrosis (CF) patients and causes a high mortality rate in both of these populations [2,3]. Moreover, antibacterial resistance of $P$. aeruginosa and consumer mistrust of synthetic additives are increasing, creating a need for new antibiotics [4,5]. Therefore, natural products will likely be one of major sources of the chemical diversity needed to thwart multiple resistance mechanisms [6]. During our search for new antibacterial compounds from a taxonomically diverse plant collection, we found that Cornus macrophylla extract had a high antibacterial activity against P. aeruginosa.

Cornus macrophylla, or GomUiMalChae in Korean, is a dogwood with large leaves that belongs to the Cormaceae family and is widespread in eastern Asia. The bark extract of C. macrophylla has anti-cancer and immunomodulatory properties [7] as well as inhibitory activity on aldose reductase [8]. However, very little is known about other biological functions of $C$. macrophylla, especially its anti-bacterial activities. Here, the isolation of the constituents of the leaves of this plant and their antibacterial effects are described. In addition, the antibacterial compound was evaluated to provide insight into the mode of action of this compound.

\section{Results and Discussion}

\subsection{Isolation of the Active Compound from C. macrophylla and Its Structure Determination}

Leaves of $C$. macrophylla (1 kg dry weight) were extracted with $80 \%$ aqueous methanol, followed by ethyl acetate, $n$-hexane, $n$-butanol or $\mathrm{H}_{2} \mathrm{O}$ (Figure 1). Among these extracts, the ethyl acetate extract showed the best antibacterial activities against $P$. aeruginosa (Table 1). Therefore, the ethyl acetate extract was solvent partitioned between chloroform, ethyl acetate and $80 \%$ methanol, after which the concentrated ethyl acetate soluble fraction was purified by silica gel column chromatography and subjected to preparative HPLC. Among the 12 fractions, fractions 1, 4, 6, 8, and 11 showed antibacterial activities (Table 2). In addition, fractions 8 and 11 showed stronger activities and the HPLC analysis of these fractions revealed the presence of flavonoids. Based on comparison with authentic standards, these compounds were identified as quercitrin and afzelin (Figure 2). This represents the first time afzelin was isolated from $C$. macrophylla.

To elucidate the antibacterial activity of quercitrin and afzelin isolated from $C$. macrophylla against P. aeruginosa, we used the disk diffusion method. Gentamycin was employed as a positive control. While quercitrin was found to have no antibacterial activity against $P$. aeruginosa, afzelin showed significant antibacterial activity; however, its activity was less potent than that of gentamycin (data not shown). 
Figure 1. Isolation of compounds $\mathbf{1}$ and $\mathbf{2}$ from the leaves of Cornus macrophylla.

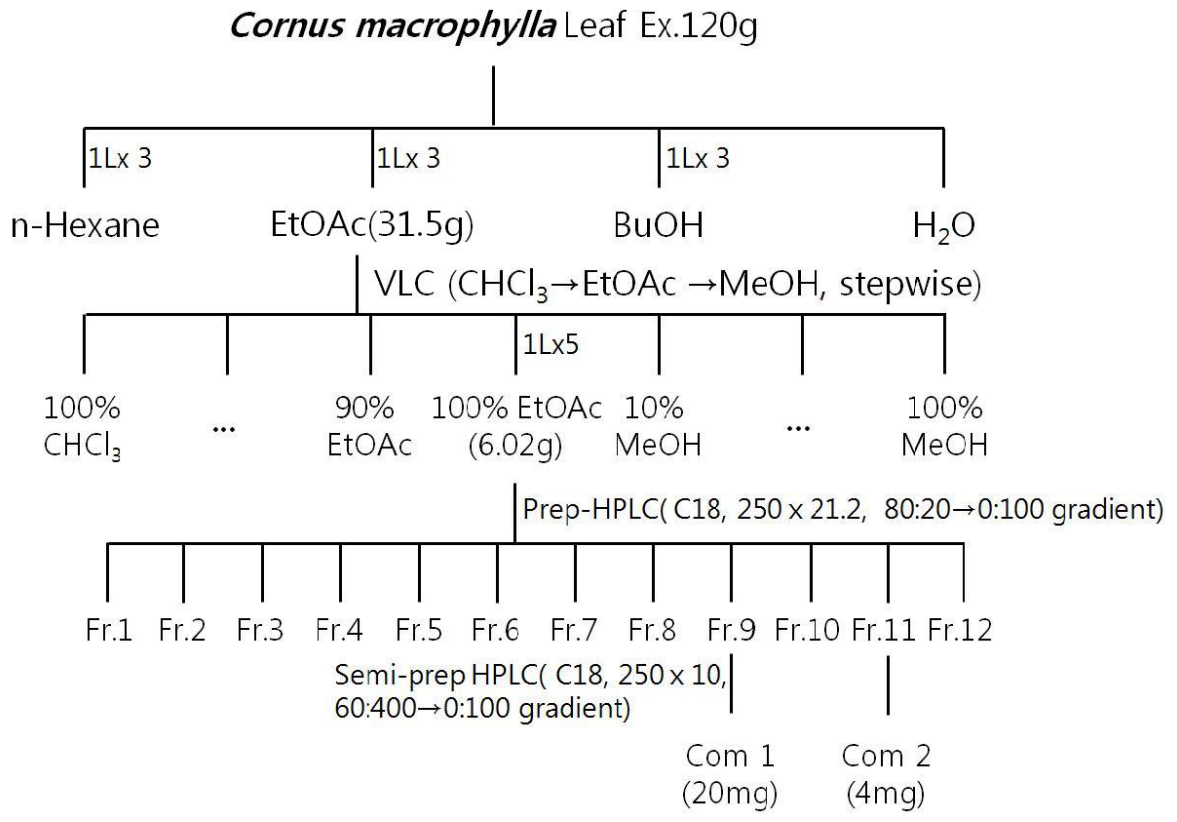

Table 1. Antibacterial activities of each solvent phase obtained from the extract of the leaves of Cornus macrophylla on the growth of P. aeruginosa.

\begin{tabular}{ccc}
\hline Fraction & Amount $(\boldsymbol{\mu g} / \mathbf{d i s c})$ & Clear Zone $(\mathbf{m m})$ \\
\hline$n$-Hexane layer & 500 & N.A. \\
EtOAc layer & 500 & 15 \\
$n$-BuOH layer & 500 & 13.1 \\
$\mathrm{H}_{2} \mathrm{O}$ layer & 500 & N.A. \\
Gentamicin & 10 & 16 \\
\hline
\end{tabular}

N.A.: No activity.

Table 2. Antibacterial activity of fractions isolated by preparative HPLC against $P$. aeruginosa.

\begin{tabular}{ccc}
\hline Fraction NO. & Amount $(\boldsymbol{\mu g} / \mathbf{d i s c})$ & Clear Zone $(\mathbf{m m})$ \\
\hline 1 & 100 & 12.5 \\
2 & 100 & N.A. \\
3 & 100 & N.A. \\
4 & 100 & 12 \\
5 & 100 & N.A. \\
6 & 100 & 12.5 \\
7 & 100 & N.A. \\
8 & 100 & 15 \\
9 & 100 & N.A. \\
10 & 100 & N.A. \\
11 & 100 & 13 \\
12 & 100 & N.A. \\
Gentamicin & 10 & 16 \\
\hline
\end{tabular}

N.A.: No activity. 
Figure 2. Structures of quercitrin and afzelin isolated from Cornus macrophylla. The arrow is the HMBC of quercitrin and afzelin.

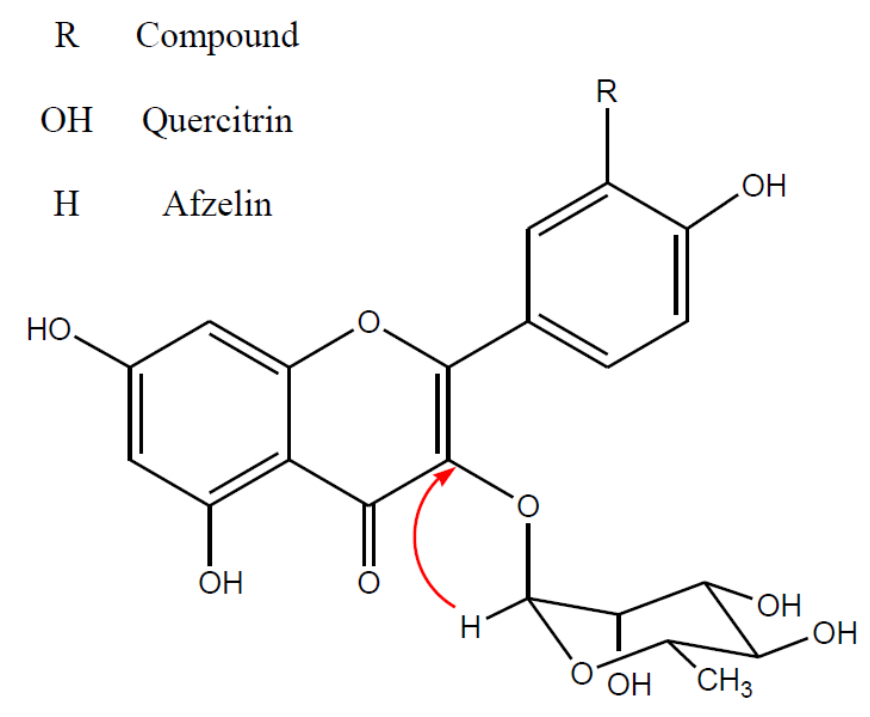

\subsection{Antimicrobial Activity of Isolated Compound against Pseudomonas aeruginosa}

The antibacterial activities of both afzelin and quercitrin were further evaluated by determining the minimum inhibitory concentration (MIC), which is the lowest concentration at which there is no growth. The MIC values of afzelin and quercitrin were determined using a two-fold serial dilution method. As shown in Table 3, while quercitrin did not inhibit the growth of Pseudomonas aeruginosa at any of the tested concentrations, while afzelin inhibited the growth of Pseudomonas aeruginosa at $31 \mu \mathrm{g} / \mathrm{mL}$, indicating that the MIC of afzelin is $31 \mu \mathrm{g} / \mathrm{mL}$. In addition, kaempferol, an aglycone of afzein, showed antibacterial activities and a MIC of $59 \mu \mathrm{g} / \mathrm{mL}$. That is, quercitrin (afzelin with $\mathrm{OH}$ at C3) showed no antibacterial activity and kaempferol (afzelin without a rhamnose group) showed less potent antibacterial activity than afzellin. The MIC of gentamicin, a positive control, was also found to be $4 \mu \mathrm{g} / \mathrm{mL}$. These results suggest that the hydroxyl group at $\mathrm{C} 3$ of the C-ring of the flavone skeleton and rhamnose group may be involved in antibacterial activity. More specifically, while the hydroxyl group at C3 of the C-ring of the flavone skeleton negatively affects antibacterial activity, the rhamnose group contributes positively to the antibacterial activity.

Table 3. Minimum inhibitory concentrations of quercitrin, afzelin and kaempferol.

\begin{tabular}{cc}
\hline \multirow{2}{*}{ Compound } & Bacteria Tested \\
\cline { 2 - 2 } & MIC $(\boldsymbol{\mu g} / \mathbf{m L})$ \\
\hline Quercitrin & N.A. \\
Afzelin & $\geq 31$ \\
Kaempferol & $\geq 59$ \\
Gentamicin & $\geq 5$ \\
\hline
\end{tabular}

N.A.: No activity.

In summary, afzelin isolated from $C$. macrophylla was characterized and evaluated for its antibacterial activities against $P$. aeruginosa. We found that afzelin is one of main antibacterial 
compounds of $C$. macrophylla active against $P$. aeruginosa. In addition, recent studies have reported that afzelin has several properties, including anti-inflammatory, anti-tumor, and anti-apoptotic activities [9-11]. Taken together, the data gathered in this study suggest that afzelin may be useful as a therapeutic agent or additive for $P$. aeruginosa-related diseases. In addition, the results presented here suggest that the hydroxyl group at $\mathrm{C} 3$ of the C-ring of the flavone skeleton and the rhamnose group may be important groups which regulate the antibacterial activity of afzelin against Pseudomonas aeruginosa.

\section{Experimental}

\subsection{General Methods}

NMR spectroscopic analysis data were recorded using a JEOL JNM- LA40 spectrometer (400 MHz for ${ }^{1} \mathrm{H}$ and $100 \mathrm{MHz}$ for ${ }^{13} \mathrm{C}$ ) in $\mathrm{CD}_{3} \mathrm{OD}$. The chemical shifts were reported in $\delta$ (ppm) units relative to the TMS signal and coupling constants $(J)$ in Hz. Preparative High Pressure Liquid Chromatography (HPLC) was conducted using a Prep LC 2000 instrument and a 2487 Dual $\lambda$ Absorbance detector (Waters). Silica gel (230-400 mesh, Merck) was used for column chromatography. All HPLC-grade organic solvents and bulk organic solvents were purchased from J.T. Baker (Phillipsburg, NJ, USA) and Duksan Company (Ansan, Korea).

\subsection{Plant Material}

Naturally-grown Cornus macrophylla (C. macrophylla) were collected from Jeju Island, Korea, from June to July 2007. A voucher sample was deposited at the Jeju Bio Diversity Research Institute of the Jeju Hi-Tech Industry Development Institute.

\subsection{Extraction and Isolation}

Leaves of $C$. macrophylla (1 kg dry weight) were homogenized and extracted with $80 \%$ methanol $(\mathrm{MeOH})(10 \mathrm{~L})$. The methanolic extracts $(120 \mathrm{~g})$ were then concentrated in vacuo, after which they were re-extracted with $n$-hexane $(1 \mathrm{~L} \times 3)$, ethyl acetate (EtOAc, $1 \mathrm{~L} \times 3)$, and $n$-butanol $(1 \mathrm{~L} \times 3)$. After reducing to dryness in vacuo, the EtOAc fraction $(31.5 \mathrm{~g})$ was subjected to vacuum liquid chromatography (VLC, silica-gel, $10 \times 12.6 \mathrm{~cm}$ ), eluted with $\mathrm{CHCl}_{3}$-EtOAc $(10: 0,9: 1,8: 2,7: 3,6: 4$, 5:5, 3:7, 1:9, 0:10) mixture, and then EtOAc-MeOH (4:6, 1:9, 0:10). The 100\% EtOAc fraction was then purified by preparative HPLC using a leaner gradient solvent $\mathrm{A}\left(\mathrm{H}_{2} \mathrm{O}\right)$ and solvent $\mathrm{B}(\mathrm{MeOH})$ for 90 min, during which time the gradient was increased from $10 \%$ solvent B to $100 \%$ solvent $\mathrm{B}$ in solvent $\mathrm{A}$ at a flow rate of $10 \mathrm{~mL} / \mathrm{min}$ to give twelve fractions (Fr. 1-Fr. 12). The obtained fractions were then analyzed by HPLC and FT-NMR $\left({ }^{1} \mathrm{H},{ }^{13} \mathrm{C}\right.$, DEPT, COSY, HMBC, HMQC). Although HPLC fractions 8 (compound 1) and 11 (compound 2) were isolated, only fraction 11 (compound 2) showed anti-microbial activity. Compounds $\mathbf{1}$ and $\mathbf{2}$ were concentrated in vacuo and dissolved in $10 \%$ DMSO and then used in the study. The average contents of compound $\mathbf{1}$ and compound $\mathbf{2}$ in C. macrophylla were $1.67 \%$ and $0.33 \%$, respectively. 
Compound 1. Yellow powder, ${ }^{1} \mathrm{H}-\mathrm{NMR}\left(\mathrm{CD}_{3} \mathrm{OD}\right) \delta 7.33(1 \mathrm{H}, \mathrm{d}, J=1.92 \mathrm{~Hz}, \mathrm{H}-2$ ') $7.31(1 \mathrm{H}$, dd, $\left.J=1.96,8.32 \mathrm{~Hz}, \mathrm{H}-6^{\prime}\right), 6.91\left(1 \mathrm{H}, \mathrm{d}, J=8.32 \mathrm{~Hz}, \mathrm{H}-5^{\prime}\right), 6.38(1 \mathrm{H}, \mathrm{d}, J=1.96 \mathrm{~Hz}, \mathrm{H}-8), 6.21(1 \mathrm{H}, \mathrm{d}$, $J=1.96 \mathrm{~Hz}, \mathrm{H}-6), 5.34$ (1H, d, $J=1.44 \mathrm{~Hz}, \mathrm{H}-1 "), 4.22$ (1H, dd, $J=1.72,3.2 \mathrm{~Hz}, \mathrm{H}-3 "), 3.75$ (1H, dd, $\left.J=3.2,9.26 \mathrm{~Hz}, \mathrm{H}-2^{\prime \prime}\right), 3.42$ (1H, m, H-5"), 3.35 (1H, t, $\left.J=5 \mathrm{~Hz}, \mathrm{H}-4 "\right), 0.94$ (3H, d, $J=6.12 \mathrm{~Hz}$, H-6"), ${ }^{13} \mathrm{C}-\mathrm{NMR}\left(\mathrm{CD}_{3} \mathrm{OD}\right) \delta 179.81$ (C-4), 166.17 (C-7), 163.37 (C-5), 159.48 (C-9), 158.7 (C-2), 149.98 (C-4'), 146.59 (C-3'), 136.37 (C-3), 123.1 (C-1'), 123.0 (C-6'), 117.07 (C-5'), 116.53 (C-2'), 106.01 (C-10), 103.7 (C-1"), 100.0 (C-6), 94.89 (C-8), 73.39 (C-4"), 72.25 (C-3"), 72.20 (C-2"), 72.05 (C-5"), $17.80(\mathrm{C}-6 ")$.

Compound 2. Yellow powder, ${ }^{1} \mathrm{H}-\mathrm{NMR}\left(\mathrm{CD}_{3} \mathrm{OD}\right) ; \delta 7.76\left(2 \mathrm{H}, \mathrm{d}, J=8.76 \mathrm{~Hz}, \mathrm{H}-2^{\prime}, 6\right.$ '), $6.93(2 \mathrm{H}, \mathrm{d}$, $\left.J=8.88 \mathrm{~Hz}, \mathrm{H}^{-} 3^{\prime}, 5^{\prime}\right), 6.36(1 \mathrm{H}, \mathrm{d}, J=2.2 \mathrm{~Hz}, \mathrm{H}-8), 6.19(2 \mathrm{H}, \mathrm{d}, J=2.44 \mathrm{~Hz}, \mathrm{H}-6), 5.36$ (1H, d, $J=2.2$ Hz, H-1"), 4.21 (1H, dd, J=1.7, 3.42 Hz, H-2"), 3.71 (H, m, H-3"), 3.60 (H, m, H-4"), 3.47 (H, m, H-5"), $0.91(3 \mathrm{H}, \mathrm{d}, J=5.6 \mathrm{~Hz}, \mathrm{H}-6 "),{ }^{13} \mathrm{C}-\mathrm{NMR}\left(\mathrm{CD}_{3} \mathrm{OD}\right) ; \delta 179.35$ (C-4), 166.6 (C-7), 163.2 (C-5), 161.63 (C-4'), 159.24 (C-9), 158.62 (C-2), 136.15 (C-3), 131.9 (C-2', 6'), 122.62 (C-1'), 116.54 (C-3', 5'), 105.73 (C-10), 103.5 (C-1"), 100.06 (C-6), 94.92 (C-8), 73.17 (C-4"), 72.09 (C-2"), 72.04 (C-3"), 71.92 (C-5"), $17.66(\mathrm{C}-6 ")$.

\subsection{Antimicrobial Test}

The antimicrobial activity of all fractions of C. macrophylla Leaf Ex. was evaluated using the standardized filter-paper disc-agar diffusion method, which is known as the Kirby-Bauer method [12]. P. aeruginosa was employed in this experiment. Bacterial cells were cultured at $37^{\circ} \mathrm{C}$ in Luria-Bertani broth (BD, Difco, Franklin Lakes, NJ, USA) under aerobic conditions until their growth reached a stationary phase. The plates were then inoculated with $10^{6} \mathrm{CFU} / \mathrm{mL}$ of the test micro-organism, after which filter paper discs (8 mm, ADVANTEC, Toyo Roshi Kaicha, Ltd., Tokyo, Japan) impregnated with various concentrations of the test materials were placed on the surface of the agar plates. The plates were then incubated at $37{ }^{\circ} \mathrm{C}$ for $24 \mathrm{~h}$ under aerobic conditions. Antimicrobial activity was evaluated by measuring the diameter of the growth inhibition zone. Gentamycin was used as a positive control.

\subsection{Minimum Inhibition Concentration (MIC) Test}

Bacterial cells were pre-cultured for $24 \mathrm{~h}$ at $37{ }^{\circ} \mathrm{C}$ on an agar plate under aerobic conditions, after which the cells $\left(10^{7}\right)$ were inoculated into $2 \mathrm{~mL}$ Luria-Bertani broth. Subsequently, test samples were added to $2 \mathrm{~mL}$ Luria-Bertani broth containing the bacteria and cultured for $24 \mathrm{~h}$ at $37{ }^{\circ} \mathrm{C}$ under aerobic conditions. To determine the MIC of the compounds, we employed a two-fold serial dilution method [13]. The MIC value was defined as the lowest concentration that yielded no bacterial cell growth.

\section{Conclusions}

Cornus macrophylla showed antibacterial activity against Pseudomonas aeruginosa, a leading cause of illness in immunocompromised individuals. Afzelin isolated from C. macrophylla was found to be one of main antibacterial compounds specifically against Pseudomonas aeruginosa. We found 
that a hydroxyl group and rhamnose group may operate as a negative factor and an enhancing factor, respectively, of the antibacterial activities of afzelin.

\section{Acknowledgements}

This study was supported by the BK21 Plus funded by the Ministry of Education, Korea (31Z20130012916).

\section{Author Contributions}

S.Y. Lee, J.Y. Cho and J. Lee conceived and designed the experiments; S.Y. Lee, J.Y. Cho and J. Lee performed the experiments; S.Y. Lee, J.Y. Cho and J. Lee analyzed the data; J. Lee and Y.J. So contributed reagents/materials/analysis tools; and S.Y. Lee, J.Y. Cho and J. Lee wrote the manuscript.

\section{Conflicts of Interest}

The authors declare no conflict of interest.

\section{References}

1. Dembry, L.M.; Roberts, J.C.; Schock, K.D.; Marino, S.P.; Farrel, P.A.; Andriole, V.T. Comparison of in vitro activity of trovafloxacin against gram-positive and gram-negative organisms with quinolones and beta-lactam antimicrobial agents. Diagn. Microbiol. Infect. Dis. 1998, 31, 301-311.

2. Molina, D.N.; Colón, M.; Bermúdez, R.H.; Ramírez-Ronda, C.H. Unusual presentation of pseudomonas aeruginosa infections: A review. Bol. Asoc. Med. Puerto Rico 1991, 83, 160-163.

3. Pollack, M.; Koles, N.L.; Preston, M.J.; Brown, B.J.; Pier, G.B. Functional properties of isotype-switched immunoglobulin $\mathrm{M}$ (IgM) and $\mathrm{IgG}$ monoclonal antibodies to pseudomonas aeruginosa lipopolysaccharide. Infect. Immun. 1995, 63, 4481-4488.

4. Ryu, S.; Labbe, R.G. Stimulation of the onset of sporulation of Clostridium perfringens type A by netropsin and distamycin. Curr. Microbiol. 1992, 25, 183-187.

5. Zink, D.L. The impact of consumer demands and trends on food processing. Emerg. Infect. Dis. 1997, 3, 467-469.

6. Payne, P.R.; Borlawsky, T.; Kamal, J.; Saltz, J.H. A framework for workflow-based clinical research billing disambiguation. AMIA Annu. Symp. Proc. 2007, 11, 1071.

7. Jin, L.; Han, J.G.; Ha, J.H.; Jeong, H.S.; Kim, C.H.; Kwon, M.C.; Lee, H.J.; Kang, H.Y.; Choi, G.P.; Lee, H.Y. Anticancer and immune-modulatory activities of extracts from various parts of cornus macrophylla wall. Korean J. Med. Crop Sci. 2008, 16, 349-355.

8. Kim, H.Y.; Oh, J.H. Screening of Korean forest plants for rat lens aldose reductase inhibition. Biosci. Biotechnol. Biochem. 1999, 63, 184-188.

9. Shin, S.W.; Jung, E.; Kim, S.; Kim, J.H.; Kim, E.G.; Lee, J.; Park, D. Antagonizing effects and mechanisms of afzelin against UVB-induced cell damage. PLoS One 2013, 8, e61971. 
10. Choe, K.I.; Kwon, J.H.; Park, K.H.; Oh, M.H.; Kim, M.H.; Kim, H.H.; Cho, S.H.; Chung, E.K.; Ha, S.Y.; Lee, M.W. The antioxidant and anti-inflammatory effects of phenolic compounds isolated from the root of Rhodiola sachalinensis A. BOR. Molecules 2012, 17, 11484-11494.

11. Rho, H.S.; Ghimeray, A.K.; Yoo, D.S.; Ahn, S.M.; Kwon, S.S.; Lee, K.H.; Cho, D.H.; Cho, J.Y. Kaempferol and kaempferol rhamnosides with depigmenting and anti-inflammatory properties. Molecules 2011, 16, 3338-3344.

12. Furtado, G.L.; Medeiros, A.A. Single-disk diffusion testing (Kirby-Bauer) of susceptibility of Proteus mirabilis to chloramphenicol: Significance of the intermediate category. J. Clin. Microbiol. 1980, 12, 550-553.

13. Park, J.; Lee, J.; Jung, E.; Park, Y.; Kim, K.; Park, B.; Jung, K.; Park, E.; Kim, J.; Park, D. In vitro antibacterial and anti-inflammatory effects of honokiol and magnolol against propionibacterium sp. Eur. J. Pharmacol. 2004, 496, 189-195.

Sample Availability: Samples of the compounds assayed are available from the authors.

(C) 2014 by the authors; licensee MDPI, Basel, Switzerland. This article is an open access article distributed under the terms and conditions of the Creative Commons Attribution license (http://creativecommons.org/licenses/by/3.0/). 